\title{
Effect of Polymer Molecular Mass and Structure on the Mechanical Properties of Polymer-Glass Hybrids
}

\author{
Yu Lin Lee, Daniel W. Lester, Julian R. Jones, and Theoni K. Georgiou*
}

Cite This: ACS Omega 2022, 7, 786-792

Read Online

ACCESS

Шll Metrics \& More

Article Recommendations

Supporting Information

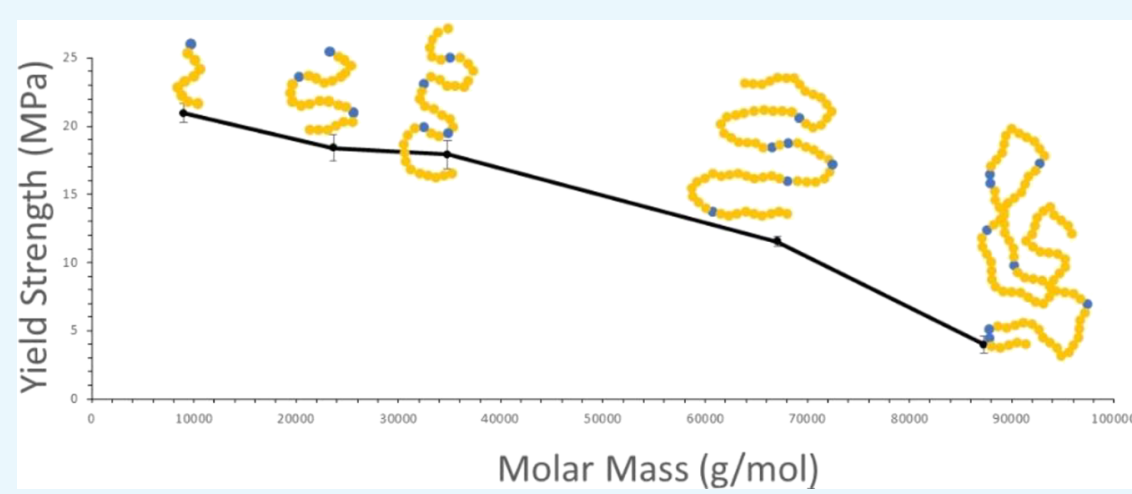

ABSTRACT: Organic-inorganic hybrid materials are a promising class of materials for tissue engineering and other biomedical applications. In this systematic study, the effect of the polymer molecular mass (MM) with a linear architecture on hybrid mechanical properties is reported. Well-defined linear poly(methyl methacrylate-co-(3-(trimethoxysilyl)propyl methacrylate)) polymers with a range of MMs of 9 to $90 \mathrm{kDa}$ and one $90 \mathrm{kDa}$ star-shaped polymer were synthesized and then used to form glasspolymer hybrids. It was demonstrated that increasing linear polymer MM decreases the resultant hybrid mechanical strength. Furthermore, a star-polymer hybrid was synthesized as a comparison and demonstrated significantly different mechanical properties relative to its linear-polymer counterpart.

\section{INTRODUCTION}

Bone defects above a certain critical size, whether caused by trauma, bone tumor surgery, infection, or other causes, can have limited regeneration capability. ${ }^{1}$ Currently, such defects are addressed using bone grafts, which involve disadvantages of the availability of patient's own bone and high invasiveness due to the requirement for multiple surgeries, bringing increased risk of infection. ${ }^{2,3}$ As such, biomaterial alternatives to grafting are of great interest. $^{4-9}$ Inorganic-organic sol-gel hybrid materials are composed of inorganic and organic conetworks that are indistinguishable above the nanoscale and have been an active area of research with potential applications toward bone regeneration. Of particular interest are type II hybrids, which have covalent bonding between the organic and inorganic components, giving rise to a single-phase material with excellent mechanical properties. $^{6-9}$ Some examples of such systems include polymer-clay hybrid nanocomposites, ${ }^{10}$ rubberorganomontmorillonite hybrid nanocomposites, ${ }^{11}$ and polymer-silica hybrids. ${ }^{9,12}$

In the context of bone regeneration, hybrids can overcome the brittleness of current inorganic synthetic bone grafts, such as bioactive glasses, which promote bone regeneration but cannot sustain cyclic loads and mitigate uneven degradation rates of the organic and inorganic components of conventional composites of particles dispersed in a polymer matrix.,13-17 Organicinorganic sol-gel hybrids have previously been synthesized using polymers that contain pendant siloxane groups such as poly(3-(trimethoxysilyl)propyl methacrylate), polyTMSPMA. These polymers have been previously synthesized through a variety of polymerization methods such as reversible additionfragmentation chain transfer polymerization, group transfer polymerization (GTP), and atom transfer radical polymerization. $^{18-23}$

In one of these studies, polymers of varying molecular masses (MMs) were fabricated, but the MM distributions were too broad to be able to correlate the mechanical properties of the hybrid with the polymer MM. ${ }^{18}$ In another interesting study, three polymers of different 3D topology were investigated, specifically branched versus star versus linear, and the resulting

Received: September 30, 2021

Accepted: December 14, 2021

Published: December 22, 2021 
Table 1. Number Average Molecular Mass $\left(M_{n}\right)$, Molecular Mass Distributions $(\boxplus)$ (See Supporting Information Figure S1 for the Gel-Permeation Chromatography (GPC) Traces), and Theoretical and Experimentally Determined Composition of All the Synthesized Copolymers

\begin{tabular}{|c|c|c|c|c|c|}
\hline \multirow[b]{2}{*}{ hybrid precursor polymer } & \multirow[b]{2}{*}{ polymer chemical structure } & \multirow[b]{2}{*}{$M_{\mathrm{n}}(\mathrm{g} / \mathrm{mol})$} & \multirow[b]{2}{*}{ dispersity $(\nexists)$} & \multicolumn{2}{|c|}{ mol \% TMSPMA } \\
\hline & & & & theo. & ${ }^{1} \mathrm{H}-\mathrm{NMR}$ \\
\hline Linear-9 K & $\mathrm{MMA}_{81}-c o-\mathrm{TMSPMA}_{4}$ & $8980^{a}$ & $1.15^{a}$ & 4 & 4 \\
\hline Linear-24 K & $\mathrm{MMA}_{212^{-c o}-{ }^{-T M S P M A}}$ & $23,600^{a}$ & $1.11^{a}$ & 4 & 5 \\
\hline Linear-35 K & $\mathrm{MMA}_{313}-$ co-TMSPMA 14 & $34,800^{a}$ & $1.10^{a}$ & 4 & 4 \\
\hline Linear-67 K & $\mathrm{MMA}_{603}-c o-\mathrm{TMSPMA}_{27}$ & $67,100^{a}$ & $1.13^{a}$ & 4 & 4 \\
\hline Linear-87 K & $\mathrm{MMA}_{785^{-}}$co-TMSPMA 35 & $87,300^{a}$ & $1.14^{a}$ & 4 & 4 \\
\hline Star-90 K & $\mathrm{MMA}_{806}-\mathcal{c o}-\mathrm{TMSPMA}_{36}-\mathrm{Star}$ & $89,700^{b}$ & $1.21^{b}$ & 4 & 4 \\
\hline
\end{tabular}

${ }^{a}$ Determined using a GPC system with a refractive index (RI) detector, in THF, calibrated using poly(methyl methacrylate) standards. ${ }^{b}$ Determined using the RI detector within a triple detector GPC system.

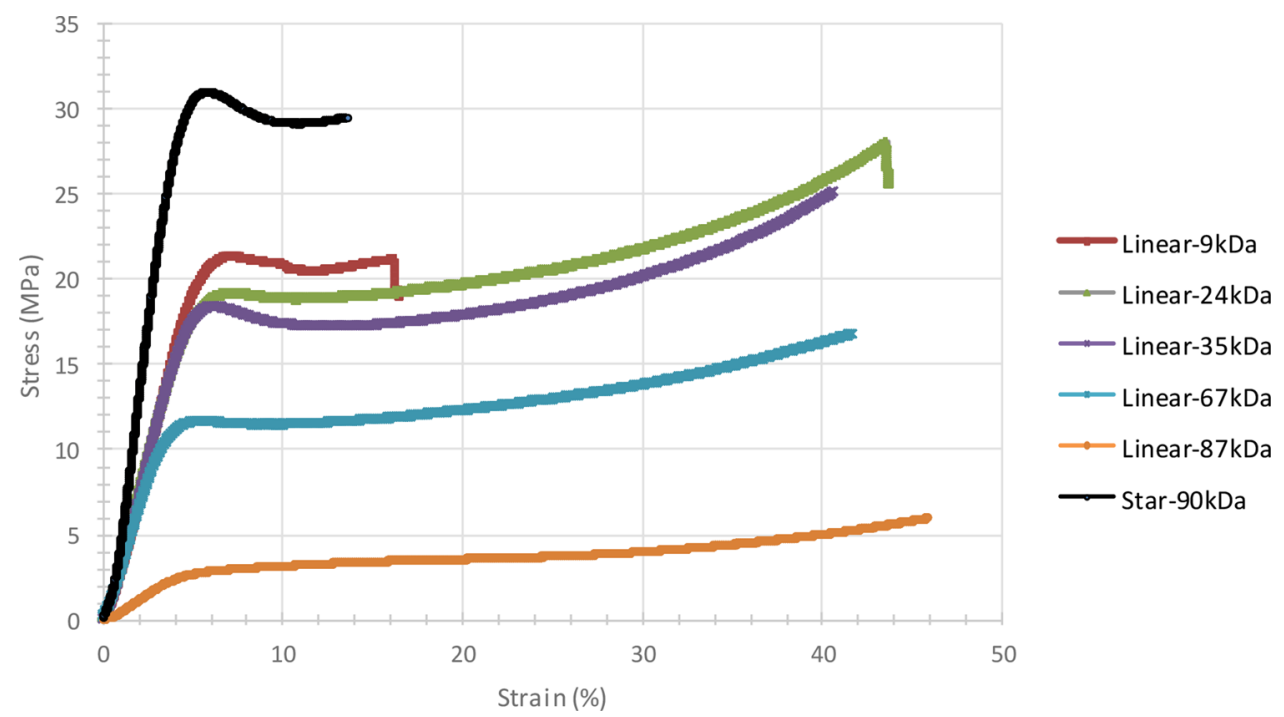

Figure 1. Characteristic stress-strain curves of hybrid materials made from different MM linear polymers and one star-polymer hybrid.

hybrids' mechanical properties varied significantly. ${ }^{20}$ However, the MMs of the three polymers studied were not kept constant.

The aim of this study was to systematically investigate the effect of the linear polymer MM on glass/polymer hybrid mechanical properties through a comparative study of hybrid biomaterials produced from different MM linear polymers synthesized through one-pot GTP. A single set of hybrid samples produced from a star polymer with similar chemistry and MM to the largest linear polymer is also analyzed and compared. To the best of our knowledge, this is the first study where the $\mathrm{MM}$ effect is systematically investigated using polymers with narrow MMD as most published studies on TMSPMA-based polymers investigated polymers with broad MMD, no reported MMD, or they do not vary the MM. ${ }^{18-25}$ Thus, this work can serve as a guide for tailoring the mechanical properties of such systems in a variety of applications.

In this study, all copolymers of $\mathrm{p}$ (MMA-co-TMSPMA) were successfully synthesized via GTP with the targeted molar ratio of monomers, specifically $\sim 4 \mathrm{~mol} \%(\sim 10 \mathrm{wt} \%)$ of TMSPMA (see Table 1), confirmed by proton nuclear magnetic resonance spectroscopy ( ${ }^{1} \mathrm{H}-\mathrm{NMR}$ ) (see Supporting Information Figure S2 for the nuclear magnetic resonance (NMR) spectra). This ratio was chosen because it was proven from previous studies that a high TMSPMA content results in brittle and easily faulted samples. ${ }^{18}$ See the Experimental Methods section at the bottom for details.

\section{RESULTS AND DISCUSSION}

To investigate the effects of polymer MM on the subsequent hybrid material's mechanical properties, five different MM linear copolymers were synthesized, while one star-shaped polymer was produced, using the same monomer units, monomer molar ratios, and with the same overall $\mathrm{MM}$ as the largest linear copolymer that was synthesized $(90 \mathrm{~kg} / \mathrm{mol}$ in total for the starshaped polymer with $\sim 9 \mathrm{kDa}$ arms vs $\sim 90 \mathrm{kDa}$ linear copolymer). The polymers were synthesized through a similar process to previous studies involving GTP. ${ }^{26,27}$ In this case, for the statistical linear copolymers, the polymerization catalyst, solvent, and monomers were syringed into a round-bottom flask, and then the initiator was added to start the reaction. For the star-shaped copolymer, an arm-first one-pot synthesis approach was used where the same process as above was used to synthesize the linear copolymer arms, and then the still active linear polymers were then crosslinked using ethylene glycol dimethacrylate (EGDMA) as the crosslinking agent. The syntheses of all linear copolymers were terminated at $20 \mathrm{~min}$, while the star-shaped polymer took a total of $40 \mathrm{~min}$ with $20 \mathrm{~min}$ for each stage. More details of the experimental procedure are provided after the conclusion.

Table 1 shows the number average MMs $\left(M_{n}\right)$ and dispersity (Đ) values of all the synthesized polymers. The $M_{\mathrm{n}}$ values for the linear copolymers ranged between 8980 and 87,300 Da to allow investigation of the effect of MM on the hybrids' properties. The 
a: Yield Strength vs Polymer Molecular Mass \& Structure

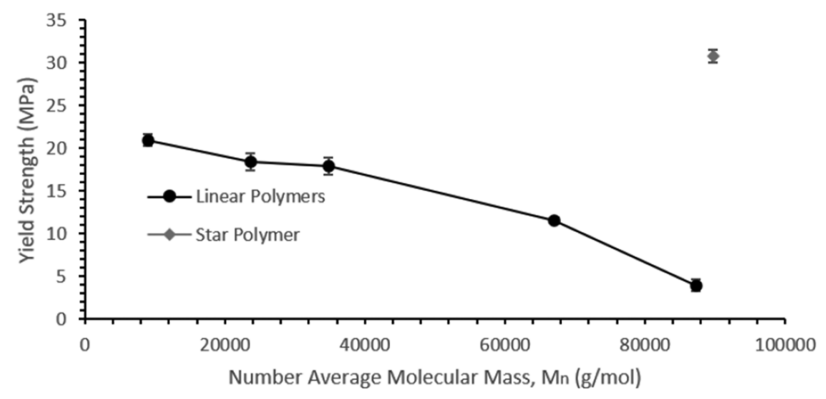

c: Young's Modulus vs Polymer Molecular Mass \&

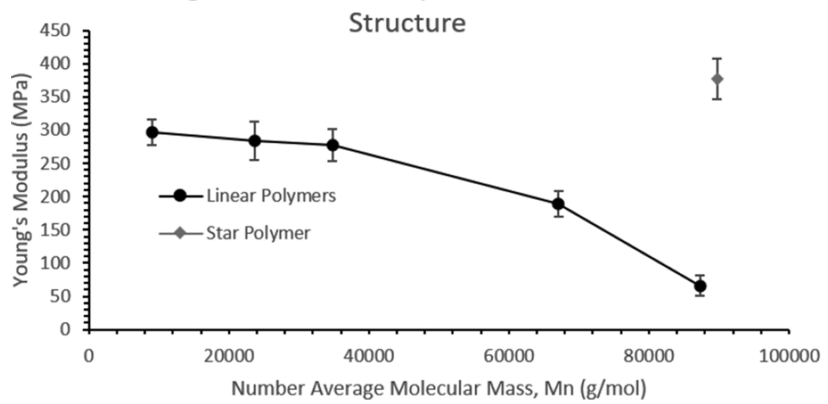

b: Yield Strain vs Polymer Molecular Mass \& Structure

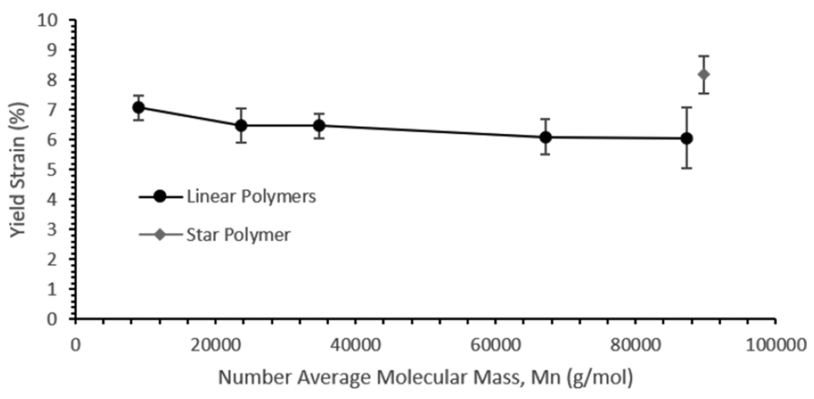

d: Resilience vs Polymer Molecular Mass \& Structure

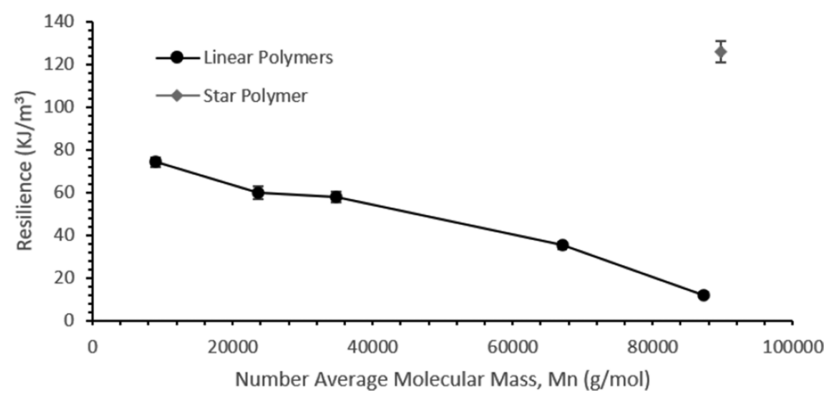

Figure 2. Hybrid mechanical properties as a function of MM for hybrids made with linear and star polymers: (a) Yield strength; (b) yield strain; (c) Young's Modulus; (d) resilience.

Table 2. Hybrid Compression Test and TGA Results

\begin{tabular}{cccccc} 
hybrid & yield strength $(\mathrm{MPa})$ & yield strain $(\%)$ & resilience $\left(\mathrm{kJ} / \mathrm{m}^{3}\right)$ & $E(\mathrm{MPa})$ & residual mass $(\mathrm{wt} \%)$ \\
Linear-9 K & $21.0 \pm 0.70$ & $7.06 \pm 0.39$ & $74.1 \pm 2.4$ & $297 \pm 19$ & 15 \\
Linear-24 K & $18.4 \pm 0.95$ & $6.48 \pm 0.57$ & $59.7 \pm 3.0$ & $284 \pm 29$ & 10 \\
Linear-35 K & $17.9 \pm 1.0$ & $6.46 \pm 0.42$ & $57.9 \pm 2.5$ & $277 \pm 24$ & 12 \\
Linear-67 K & $11.5 \pm 0.36$ & $6.09 \pm 0.59$ & $35.1 \pm 1.8$ & $189 \pm 19$ & 14 \\
Linear-87 K & $4.00 \pm 0.63$ & $6.06 \pm 1.0$ & $12.1 \pm 1.4$ & $66 \pm 15$ & 17 \\
Star-90 K & $30.8 \pm 0.72$ & $8.17 \pm 0.63$ & $126 \pm 5.1$ & $377 \pm 30$ & 12 \\
\hline
\end{tabular}

overall $M_{\mathrm{n}}$ of the star polymer is close to that of the highest $M_{\mathrm{n}}$ linear copolymer at $89,700 \mathrm{Da}$. The dispersity values were low, with $Ð$ less than 1.2 for the linear copolymers and just over 1.2 for the star-shaped polymers, indicating well-defined polymers. To the best of our knowledge, this is also the first time that GTP has been used to synthesize methacrylate-based linear copolymers of such high MM and low $Ð$-with 2-(tigloyloxy)ethyl methacrylate (TiEMA)- $b$-EGDMA at $67.8 \mathrm{kDa}$ and a $Ð$ of 1.71 being one of the largest previously reported controlled GTP synthesized methacrylate copolymers. ${ }^{27}$ The $M_{n}$ values of the largest linear copolymer and of the star-shaped polymer were similar ( $<3 \%$ difference), enabling comparison between the mechanical properties of their hybrids.

All the synthesized copolymers were made into polymerglass hybrid materials through the sol-gel process reported in previous studies. ${ }^{19-23}$ In brief, tetraethyl orthosilicate (TEOS) was used as the silicate source and was combined with water and hydrochloric acid $(\mathrm{HCl})$ as a catalyst to undergo hydrolysis for 1 h. The synthesized polymer was then added to the reaction pot for polymerization with the hydrolyzed TEOS monomers. The resulting sol-gel was then poured into a screw top poly(methyl pentene) mold, then aged, and dried in an oven for 2 weeks for the hybrid to form and the solvents to evaporate, resulting in hybrids of approximately $15 \mathrm{~mm}$ length and $8 \mathrm{~mm}$ in diameter.
Six samples of cylindrical shaped hybrids suitable for uniaxial compression tests were produced for each different polymer (See Supporting Information Figure S4 for sample pictures). These were then compression tested according to the ISO 604:2003 standard for plastics compression tests where samples were tested at $5 \mathrm{~mm} / \mathrm{min}$ without preloading. Thermogravimetric analysis (TGA) was used to characterize their organic to inorganic compositions (see Supporting Information Figure S3). The synthesized hybrids all had similar residual mass (silica glass) after thermal degradation at around $15 \mathrm{wt} \%$, which was similar to the nominal composition.

All the different hybrids had clear elastic deformation and plastic deformation regimes that facilitated yield point identification (Figure 1). Hybrids synthesized from polymers of different MMs performed differently during uniaxial compression tests. The key finding here is that as the MM for the linear copolymers increased, yield strength of the hybrids decreased (Figure 2). Hybrids made from the $9 \mathrm{kDa} M_{\mathrm{n}}$ linear copolymers averaged $21 \mathrm{MPa}$ in yield strength (Table 2), whereas the hybrids made from $87 \mathrm{kDa} M_{\mathrm{n}}$ linear copolymers only averaged $4 \mathrm{MPa}$ in yield strength. The decrease in yield strength with increasing linear copolymer $M_{n}$ is consistent and clearly observed and has low error. The strain behavior was similar for all the hybrids synthesized from different $M_{\mathrm{n}}$ at 6 to $7 \%$ strain. As a result, Young's moduli and resilience (area under 


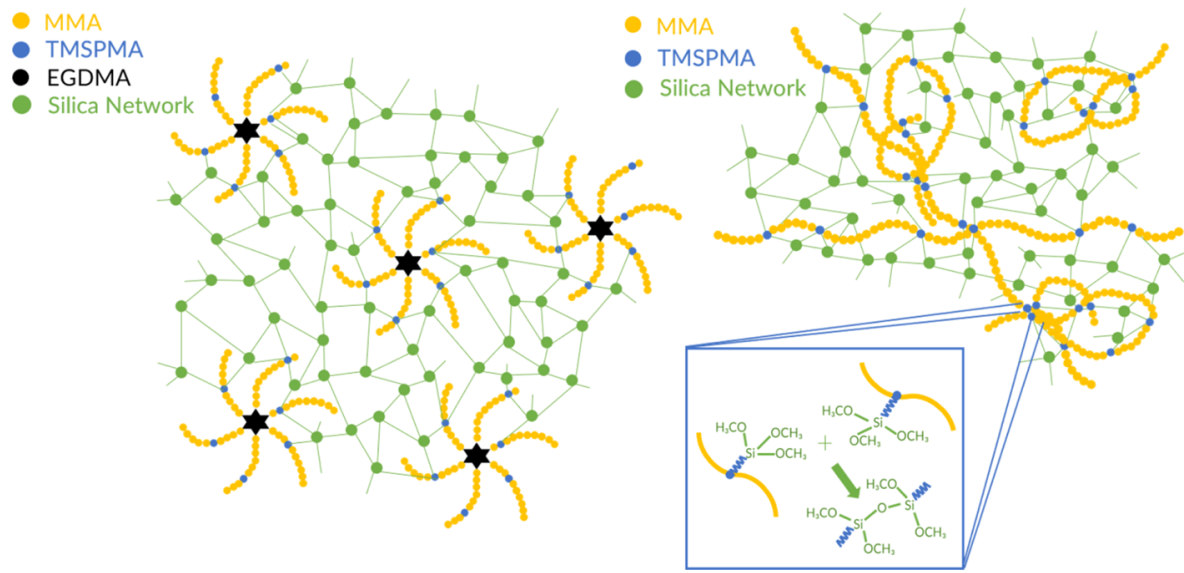

Figure 3. (Left) schematic representation of star-polymer integration into a hybrid silica network. (Right) schematic representation of linear polymers into a hybrid silica network.

the stress-strain curve up to the yield point) also decreased as $M_{\mathrm{n}}$ increased.

Interestingly, the $\sim 90 \mathrm{kDa} M_{\mathrm{n}}$ star-shaped copolymer exhibited the highest mechanical performance overall in terms of yield strength, yield strain, Young's modulus, and resilience. Hybrids synthesized from the star-shaped copolymer outperformed even hybrids synthesized from $9 \mathrm{kDa} M_{\mathrm{n}}$ linear copolymers. The improvement is even more striking compared to hybrids synthesized from linear copolymers of similar $M_{n}$ to the star-shaped polymer. While the star-shaped polymer hybrid had a yield strength of around $31 \mathrm{MPa}$, the $87 \mathrm{~K}$ linear copolymer hybrid had a yield strength of only $4 \mathrm{MPa}$ (Figure 2). This is an order of magnitude difference in yield strength and Young's modulus between the star-shaped copolymer hybrid and the linear copolymer hybrid.

These trends may be explained by inherent star-polymer morphologies and enhanced self and cooperative diffusion properties of star-polymer solutions. ${ }^{27,28}$ In particular, previous research has shown that well-defined star polymers have corecorona morphologies where star-polymer arms tend to have unequal mass distribution resulting in more worm-like coiling rather than compact random coiling for the arms. ${ }^{29}$ Previous studies have also shown that star polymers have a longer range order in solution than their linear counterparts. ${ }^{27,29}$ Furthermore, the viscosity of a star-polymer solution mostly increases relative to its arm $\mathrm{MM}$ rather than its overall $\mathrm{MM}$, and they overall have lower intrinsic viscosity, radius of gyration, and enhanced osmotic pressure at the same overall MM than their linear counterparts. ${ }^{28-32}$ In combination, these suggest that when forming hybrids, star polymers may have more uniform interactions with TEOS silica sources during synthesis, as the pendant siloxane groups on TMSPMA units along the starpolymer arms are more likely to react with TEOS silica sources rather than with each other and better integrate the polymers into the overall silica network. Star polymers would also diffuse more uniformly during the sol-gel process and be integrated into the resultant silica network of the hybrid material with more long-distance ordering giving rise to a more coherent hybrid network structure (see Figure 3 for a schematic representation). Higher $M_{n}$ linear copolymers have higher probability of crosslinking with themselves, because of random coil, where pendant siloxane groups form bonds with other pendant groups along the chain, rather than with the silicate network from the TEOS-based sol during hybrid synthesis. This behavior could be amplified by an inferior long-range order compared to equivalent $M_{\mathrm{n}}$ star polymers in solution and result in nonuniform integration of linear polymers into the hybrid silica network (see Figure 3 for a schematic representation of this process $).{ }^{27,28}$ The less uniform distribution of the polymeric organic component among the resultant hybrids synthesized from high $M_{\mathrm{n}}$ linear polymers may have more concentrated network defects and thereby lead to inferior mechanical performance. It is also possible that the EGDMA used as starpolymer cores-which make up just under $10 \mathrm{wt} \%$ - has some additional effect on hybrid mechanical properties that has led to this observation. Finally, the small amount $(\sim 1 \mathrm{wt} \%)$ of residual linear polymers in the star-polymer solution as an artifact of the purification process (which involved six consecutive purifications) may also have an impact on subsequent hybrid mechanical properties. The effect of mixing linear polymers with star polymers on the resultant polymer-glass hybrid mechanical properties warrants further investigation.

\section{CONCLUSIONS}

Five linear methacrylate-based copolymers of different MMs and one star-shaped polymer of low dispersity were synthesized through GTP and then used as precursor materials for corresponding polymer-glass hybrids, which were fabricated through the sol-gel process. The resultant hybrid materials from linear copolymers showed decreasing yield strength, Young's modulus, and resilience as the MM of the polymer precursor increased. However, the star-shaped polymer-based hybrid material demonstrated superior mechanical performance compared to hybrids made from a chemically equivalent linear copolymer of similar MM.

\section{EXPERIMENTAL SECTION}

Materials. Methyl methacrylate (99\%, stabilized), 3(trimethoxysilyl)propyl methacrylate (TMSPMA, 98\%), ethylene glycol dimethacrylate (EGDMA, 98\%, contains 90-110 ppm monomethyl ether hydroquinone as the inhibitor), 1methoxy-2methyl-1-(trimethylsiloxy)propene (MTS, >90\%), 2,2-diphenyl-1-picrylhydrazyl (DPPH), activated basic alumina $(\mathrm{Al} 2 \mathrm{O} 3: \mathrm{KOH})$, neutral alumina, calcium hydride $(\mathrm{CaH} 2)$, tetrahydrofuran (HPLC-grade THF for GPC), hexane (anhydrous, 95\%), ethanol (96\%), hydrochloric acid ( $\mathrm{HCl}$, ACS reagent, $37 \%)$, TEOS (98\% reagent grade), and deuterated chloroform $(\mathrm{CDCl} 3,99.8 \%)$ were purchased from Sigma- 
Aldrich, United Kingdom. Tetrabutyl ammonium bibenzoic acid (TBABB) was synthesized previously in the lab and used as is.

Linear-Polymer Synthesis through GTP. First, all monomers were purified through two columns of basic alumina, while TMSPMA was passed through one column of neutral alumina. After purification, the monomers were placed in roundbottom flasks where calcium hydride $\left(\mathrm{CaH}_{2}\right)$ was added. Additionally, DPPH was added to all purified monomers as an inhibitor. The round-bottom flasks were then sealed with a stirrer bar, a rubber septum each, and wrapped with parafilm. The purified monomers were then placed in a fridge for $\mathrm{CaH} 2$ to absorb any residual moisture for a minimum of $72 \mathrm{~h}$ before the next steps. MTS was used as is from the manufacturer, taken directly from the fridge when needed.

After sufficient drying with $\mathrm{CaH} 2$, the monomers were distilled via vacuum distillation. Upon distillation, the Schlenk tubes were then purged with argon gas, sealed with rubber septa, wrapped with parafilm, and placed in the fridge to await polymerization.

Syntheses of the linear block copolymers were carried out through GTP (Figure 4). As an example, the synthesis of the

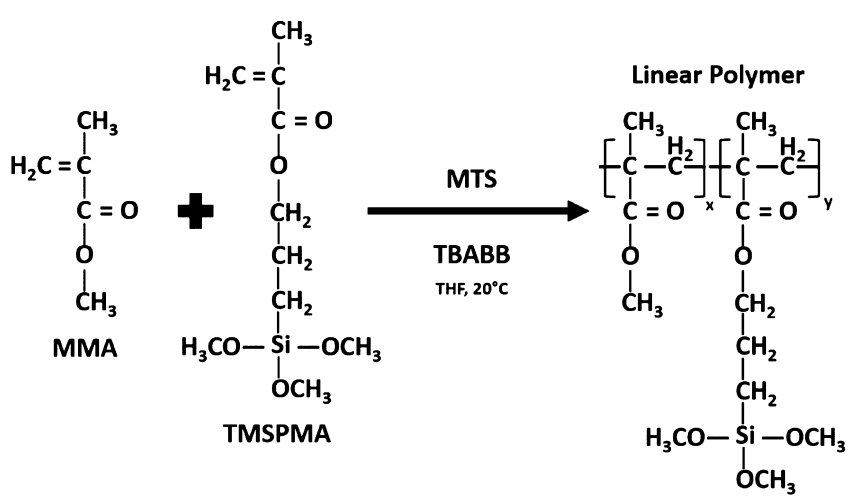

Figure 4. GTP of MMA-co-TMSPMA copolymers from monomers.

targeted $8 \mathrm{kDa}, 90$ wt \% MMA - 10 wt \% TMSPMA linear polymer is provided in brief: $10 \mathrm{mg}$ of TBABB catalyst was added into a $250 \mathrm{~mL}$ round-bottom flask which was then sealed with a rubber septum. Argon was used to purge and displace the air in the flask to eliminate moisture. THF polymer solvent $(47.99 \mathrm{~mL})$ was then syringed into the sealed flask. Then, 12.06 $\mathrm{mL}$ of MMA monomer $(0.113 \mathrm{~mol}, 11.34 \mathrm{~g})$ and $1.21 \mathrm{~mL}$ of
TMSPMA monomer $(0.005 \mathrm{~mol}, 1.26 \mathrm{~g})$ were syringed into the flask. The MTS initiator $(0.32 \mathrm{~mL})$ was quickly syringed into the sealed flask and temperature began to increase, indicating a reaction. The temperature increased from 23.8 to $40.6{ }^{\circ} \mathrm{C}$ and stopped increasing which indicated the termination of the polymerization. Two $1 \mathrm{~mL}$ samples of the polymer were extracted using a syringe and placed in two separate small glass vials for later use in GPC and ${ }^{1} \mathrm{H}-\mathrm{NMR}$ spectroscopy. At the 20 min mark since the start of polymerization, two drops of methanol were added to both sample vials, and $1 \mathrm{~mL}$ of THF solvent was added to the GPC sample vial. Both samples in the vials were then thoroughly mixed using fresh disposable syringes. All linear polymers were synthesized in the same fashion but with different amounts of reagents.

Star-Polymer Synthesis Arm-First Approach through GTP. Star-polymer synthesis is carried out in an arm-first fashion where linear polymer arms were synthesized using the same method as outlined in the previous section (Figure 5). To form the star polymers, $1.49 \mathrm{~mL}$ of EGDMA crosslinker $(0.008 \mathrm{~mol}$, $1.56 \mathrm{~g}$ ) was syringed into the sealed flask with active $8 \mathrm{kDa}$ linear polymers, and the temperature increased from 25.6 to 27.7 indicating the start and end of the crosslinking reaction. At the 20 min mark since the start of polymerization the reaction was terminated with methanol, two star-polymer samples were collected in the same fashion as previously for GPC and ${ }^{1} \mathrm{H}$ NMR.

Star-Polymer and Linear-Polymer Purification. To purify polymers, large glass beakers $(800 \mathrm{~mL}+)$ were filled with four parts ethanol and one part hexane with a stirrer bar added. These were placed on their respective stirrer plates. Aluminum foil was used to cover each beaker, two holes were then punctured, and then using a needle connected to the argon line, the beaker was filled with argon gas for $3 \mathrm{~min}$. While the hexane-ethanol solution was stirred, the polymers to be purified were poured into the prepared beakers directly from their respective round-bottom flasks while dissolved in THF. Once polymers visibly precipitate to the bottom of the beakers, the liquid content of the beakers was carefully poured out into a spare beaker until only the precipitated polymers remain in the beakers. These were then redissolved in THF, filled with argon gas, and sealed with aluminum foil. Upon fully dissolving, GPC samples were taken with the same steps as sample collection during polymerization. The GPC samples are analyzed to see if any undesirable peaks remained. The purification process was repeated until as much of the undesired peak was removed as

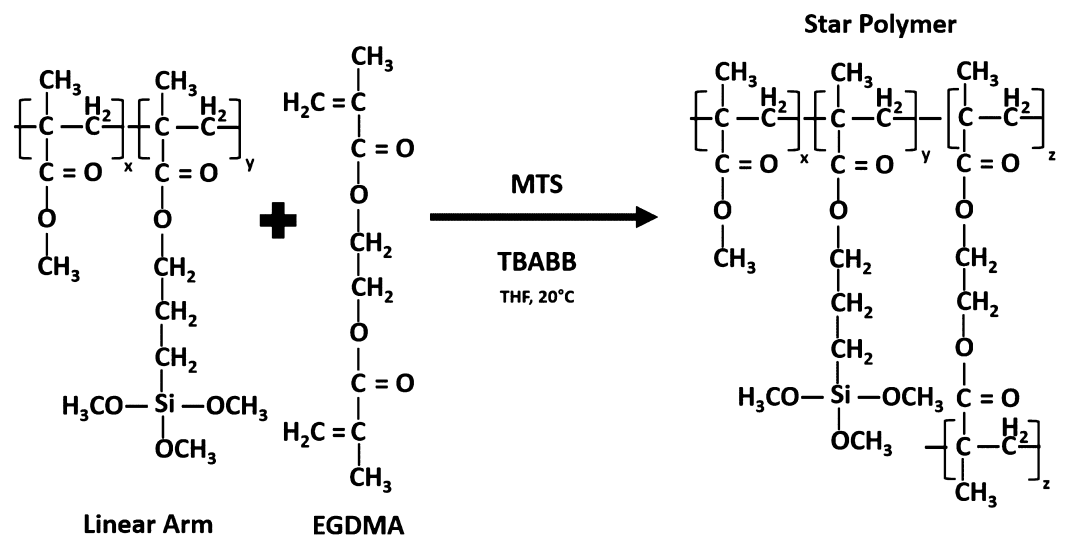

Figure 5. GTP of MMA-co-TMSPMA-star star polymers from the linear arm and EGDMA. 
possible, the process was stopped when additional purification resulted in no difference in polymer peaks.

Gel-Permeation Chromatography. First the samples previously taken during polymer syntheses were filtered by syringing them through Millex-LCR filters. The GPC solvent used was pure HPLC-grade THF calibrated using prefabricated reference poly(methyl methacrylate) samples of 2000, 4000, $8000,20,000,50,000$, and 100,000 Da. These were calibrated on a SECurity GPC system with the Polymer Standards Service SDV analytical M column (SDA083005LM). Both linear polymers and star polymers were analyzed with a RI detector. For star polymers, additional analysis was performed through triple detection using combined RI, light scattering, and viscometry. For star polymers, additional analyses on polymer branching were performed by plotting a Mark-Houwink plot of molecular weight vs intrinsic viscosity to confirm branching and formation of star polymers.

Hybrid Synthesis through the Sol-Gel Process. Syntheses of the organic-inorganic hybrid materials were carried out through the sol-gel process. A representative procedure for a $90 \mathrm{wt} \%$ organic content hybrid is outlined here. First, hydrolysis of TEOS was started by the addition of 2.989 $\mathrm{mL}$ TEOS, $0.965 \mathrm{~mL}$ deionized water, and $0.322 \mathrm{~mL}$ of $1 \mathrm{M} \mathrm{HCl}$ into a polytetrafluoroethylene (PTFE) stirring container in a molar ratio of $1: 4: 0.00016$ respectively (water to $\mathrm{HCl}$ is a volume ratio of 4 to 1 ). A stirrer bar was added, the container sealed, then the solution was left to react for an hour to fully hydrolyze. While the TEOS was undergoing hydrolysis, dissolved polymer containing $9.25 \mathrm{~g}$ of the polymer was put under a rotary evaporator until fully dry, then immediately dissolved in THF at a concentration of $1 \mathrm{~g} / \mathrm{mL}$. Once the TEOS fully hydrolyzed, the prepared polymer was poured into the TEOS container then resealed. The reaction was allowed to proceed for up to $3 \mathrm{~min}$, and then the sol-gel was poured into prepared PTFE molds and then placed in a PTFE container with a lid. The container with the mold was then sealed and placed in a 40 degrees Celsius oven to age. The aging process took 2 weeks.

Proton Nuclear Magnetic Resonance Spectroscopy. ${ }^{1} \mathrm{H}-\mathrm{NMR}$ spectroscopy was used to confirm the composition of polymers using deuterated chloroform and a $400 \mathrm{MHz}$ Avance Bruker NMR spectrometer. Samples were prepared by dissolving $0.1 \mathrm{~mL}$ of a sample polymer in up to $2 \mathrm{~mL}$ of $\mathrm{CDCl} 3$, mixed well with a pipette, and then deposited in an NMR tube.

Hybrid Mechanical Tests. Mechanical properties of hybrid materials were evaluated via uniaxial compression tests conforming to the ISO 604:2003 standard for plastics compression tests. Cylindrical hybrid specimens where the height/diameter ratio is greater than 1 were synthesized for compression tests. Sanding paper was used to manually grind the specimen ends to flat and parallel states. A caliper was used to measure the height and cross-sectional width of the specimen at different orientations 5 times and then used to obtain average values for both dimensions. A Zwick 1474 instrument with a compression speed of $0.5 \mathrm{~mm} / \mathrm{min}$ and $10 \mathrm{kN}$ load cell was used for testing. The raw data obtained consisted of force applied over compression plate moving distance. These values were then used to calculate engineering stress and strain using the following formula:

$$
\text { Stress, } \sigma=\frac{F}{h \pi r^{2}}
$$

Strain, $\varepsilon=\frac{\Delta h}{h}$

Where $F$ is the force in newtons, $h$ is the sample height in $\mathrm{mm}, r$ is the sample radius, and $\Delta h$ is the compression plate movement distance in $\mathrm{mm}$. Resilience was found as area under the stressstrain curve up to the end of the linear region.

Thermogravimetric Analysis. A NETZSCH STA 449C TGA machine was used to measure the organic content of hybrid material samples. Hybrid samples were ground to a fine powder manually using a crucible and grinder. Between 10 and $30 \mathrm{mg}$ of powder were placed in platinum crucible cleaned with acetone and with prior measured mass. The samples were then heated up to $800{ }^{\circ} \mathrm{C}$ at $10{ }^{\circ} \mathrm{C} / \mathrm{min}$ with continuously supplied air to burn away organic components. The mass change over temperature is plotted, and the inorganic content was determined by the leftover mass at the end of the measurement.

\section{ASSOCIATED CONTENT}

\section{SI Supporting Information}

The Supporting Information is available free of charge at https://pubs.acs.org/doi/10.1021/acsomega.1c05424.

Materials, experimental procedures, GPC spectra, ${ }^{1} \mathrm{H}$ NMR spectra, and compression test stress-strain curves (PDF)

\section{AUTHOR INFORMATION}

\section{Corresponding Author}

Theoni K. Georgiou - Department of Materials, Imperial College London, London SW7 2AZ, U.K.; 다이.org/00000003-4474-6931; Email: t.georgiou@imperial.ac.uk

\section{Authors}

Yu Lin Lee - Department of Materials, Imperial College London, London SW7 2AZ, U.K.

Daniel W. Lester - Polymer Characterisation Research Technology Platform, University of Warwick, Coventry CV4 7AL, U.K.

Julian R. Jones - Department of Materials, Imperial College

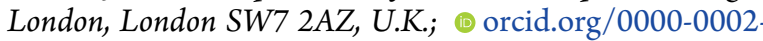
2647-8024

Complete contact information is available at:

https://pubs.acs.org/10.1021/acsomega.1c05424

\section{Author Contributions}

The manuscript was written through contributions of all authors. All authors have given approval to the final version of the manuscript.

Notes

The authors declare no competing financial interest.

\section{REFERENCES}

(1) Reichert, J. C.; Saifzadeh, S.; Wullschleger, M. E.; Epari, D. R.; Schütz, M. A.; Duda, G. N.; Schell, H.; van Griensven, M.; Redl, H.; Hutmacher, D. W. The Challenge of Establishing Preclinical Models for Segmental Bone Defect Research. Biomaterials 2009, 30, 2149-2163.

(2) Lu, N.; Lu, Y.; Liu, S.; Jin, C.; Fang, S.; Zhou, X.; Li, Z. TailorEngineered POSS-Based Hybrid Gels for Bone Regeneration. Biomacromolecules 2019, 20, 3485-3493.

(3) Mohammadi, M.; Alibolandi, M.; Abnous, K.; Salmasi, Z.; Jaafari, M. R.; Ramezani, M. Fabrication of Hybrid Scaffold Based on Hydroxyapatite-Biodegradable Nanofibers Incorporated with Lip- 
osomal Formulation of BMP-2 Peptide for Bone Tissue Engineering. Nanomed.: Nanotechnol. Biol. Med. 2018, 14, 1987-1997.

(4) Gomide, V. S.; Zonari, A.; Ocarino, N. M.; Goes, A. M.; Serakides, R.; Pereira, M. M. In Vitro and in Vivo Osteogenic Potential of Bioactive Glass-PVA Hybrid Scaffolds Colonized by Mesenchymal Stem Cells. Biomed. Mater. 2012, 7, No. 015004.

(5) Marins, N. H.; Lee, B. E. J. E.; e Silva, R. M.; Raghavan, A.; Villarreal Carreño, N. L.; Grandfield, K. Niobium Pentoxide and Hydroxyapatite Particle Loaded Electrospun Polycaprolactone/Gelatin Membranes for Bone Tissue Engineering. Colloids Surf. B Biointerfaces 2019, 182, No. 110386

(6) Sanchez, C.; Julián, B.; Belleville, P.; Popall, M. Applications of Hybrid Organic-Inorganic Nanocomposites. J. Mater. Chem. 2005, 15, 3559-3592.

(7) Mammeri, F.; Le Bourhis, E.; Rozes, L.; Sanchez, C. Mechanical Properties of Hybrid Organic-Inorganic Materials. J. Mater. Chem. 2005, 15, 3787-3811.

(8) Nicole, L.; Boissière, C.; Grosso, D.; Quach, A.; Sanchez, C. Mesostructured Hybrid Organic-Inorganic Thin Films. J. Mater. Chem. 2005, 15, 3598-3627.

(9) Jones, J. R. Review of Bioactive Glass: From Hench to Hybrids. Acta Biomater. 2013, 9, 4457-4486.

(10) Laus, M.; Francescangeli, O.; Sandrolini, F. New hybrid nanocomposites based on an organophilic clay and poly (styrene-bbutadiene) copolymers. J. Mater. Res. 1997, 12, 3134-3139.

(11) Wang, S.; Long, C.; Wang, X.; Li, Q.; Qi, Z. Synthesis and properties of silicone rubber/organomontmorillonite hybrid nanocomposites. J. Appl. Polym. Sci. 1998, 69, 1557-1561.

(12) Hajji, P.; David, L.; Gerard, J. F.; Pascault, J. P.; Vigier, G. Synthesis, structure, and morphology of polymer-silica hybrid nanocomposites based on hydroxyethyl methacrylate. J. Polym. Sci., Part B: Polym. Phys. 1999, 37, 3172-3187.

(13) Jones, J. R.; Ehrenfried, L. M.; Hench, L. L. Optimising Bioactive Glass Scaffolds for Bone Tissue Engineering. Biomaterials 2006, 27, 964-973.

(14) Fu, Q.; Saiz, E.; Tomsia, A. P. Direct Ink Writing of Highly Porous and Strong Glass Scaffolds for Load-Bearing Bone Defects Repair and Regeneration. Acta Biomater. 2011, 7, 3547-3554.

(15) Doiphode, N. D.; Huang, T.; Leu, M. C.; Rahaman, M. N.; Day, D. E. Freeze Extrusion Fabrication of 13-93 Bioactive Glass Scaffolds for Bone Repair. J. Mater. Sci. Mater. Med. 2011, 22, 515-523.

(16) Oonishi, H.; Hench, L. L.; Wilson, J.; Sugihara, F.; Tsuji, E.; Matsuura, M.; Kin, S.; Yamamoto, T.; Mizokawa, S. Quantitative Comparison of Bone Growth Behavior in Granules of Bioglass $\AA$, A-W Glass-Ceramic, and Hydroxyapatite. J. Biomed. Mater. Res. 2000, 51, $37-46$.

(17) Fu, Q.; Saiz, E.; Tomsia, A. P. Bioinspired Strong and Highly Porous Glass Scaffolds. Adv. Funct. Mater. 2011, 21, 1058-1063.

(18) Maçon, A. L. B.; Page, S. J.; Chung, J. J.; Amdursky, N.; Stevens, M. M.; Weaver, J. V. M.; Hanna, J. V.; Jones, J. R. A Structural and Physical Study of Sol-Gel Methacrylate-Silica Hybrids: Intermolecular Spacing Dictates the Mechanical Properties. Phys. Chem. Chem. Phys. 2015, 17, 29124-29133.

(19) Chung, J. J.; Fujita, Y.; Li, S.; Stevens, M. M.; Kasuga, T.; Georgiou, T. K.; Jones, J. R. Biodegradable Inorganic-Organic Hybrids of Methacrylate Star Polymers for Bone Regeneration. Acta Biomater. 2017, 54, 411-418.

(20) Chung, J. J.; Li, S.; Stevens, M. M.; Georgiou, T. K.; Jones, J. R. Tailoring Mechanical Properties of Sol-Gel Hybrids for Bone Regeneration through Polymer Structure. Chem. Mater. 2016, 28, 6127-6135.

(21) Chung, J. J.; Jones, J. R.; Georgiou, T. K. Toward Hybrid Materials: Group Transfer Polymerization of 3-(Trimethoxysilyl) Propyl Methacrylate. Macromol. Rapid Commun. 2015, 36, 1806-1809.

(22) Chung, J. J.; Sum, B. S. T.; Li, S.; Stevens, M. M.; Georgiou, T. K.; Jones, J. R. Effect of Comonomers on Physical Properties and Cell Attachment to Silica-Methacrylate/Acrylate Hybrids for Bone Substitution. Macromol. Rapid Commun. 2017, 38, No. 1700168.
(23) Li Volsi, A.; Tallia, F.; Iqbal, H.; Georgiou, T. K.; Jones, J. R. Enzyme Degradable Star Polymethacrylate/Silica Hybrid Inks for 3D Printing of Tissue Scaffolds. Mater. Adv. 2020, 1, 3189-3199.

(24) Sun, Y.; Liu, W. Preparation and Properties of an OrganicInorganic Hybrid Materials Based on Fluorinated Block Copolymer. J. Mater. Sci. 2012, 47, 1803-1810.

(25) Yuk, H.; Zhang, T.; Lin, S.; Parada, G. A.; Zhao, X. Tough Bonding of Hydrogels to Diverse Non-Porous Surfaces. Nat. Mater. 2016, 15, 190-196.

(26) Patrickios, C. S.; Lowe, A. B.; Armes, S. P.; Billingham, N. C. ABC Triblock Polymethacrylates: Group Transfer Polymerization Synthesis of the $\mathrm{ABC}, \mathrm{ACB}$, and $\mathrm{BAC}$ Topological Isomers and Solution Characterization. J. Polym. Sci. Part A Polym. Chem. 1998, 36, 617-631.

(27) Kassi, E.; Constantinou, M. S.; Patrickios, C. S. Group Transfer Polymerization of Biobased Monomers. Eur. Polym. J. 2013, 49, 761767.

(28) Hadjichristidis, N.; Pitsikalis, M.; Iatrou, H.; Driva, P.; Sakellariou, G.; Chatzichristidi, M. Polymers with Star-Related Structures: Synthesis, Properties, and Applications; Elsevier B.V., 2012; 6.

(29) Fetters, L. J.; Kiss, A. D.; Pearson, D. S.; Ag, M.; Frankfurt, D.; Vitus, F. J. Rheological Behavior of Star-Shaped Polymers. 1993, 647654.

(30) Vlassopoulos, D.; Kapnistos, M.; Fytas, G.; Roovers, J. Dynamics of Interacting Spherical Polymer Brushes. Am. Chem. Soc. Polym. Prepr. Div. Polym. Chem. 1999, 40, 94.

(31) Rey, A.; Freire, J. J.; Bishop, M.; Clarke, J. H. R. Radius of Gyration and Viscosity of Linear and Star Polymers in Different Regimes. Macromolecules 1992, 25, 1311-1315.

(32) Ganazzoli, F.; Allegra, G.; Colombo, E.; De Vitis, M. Dynamics of Regular Star Polymers: The Intrinsic Viscosity. Macromolecules 1995, 28, 1076-1084.

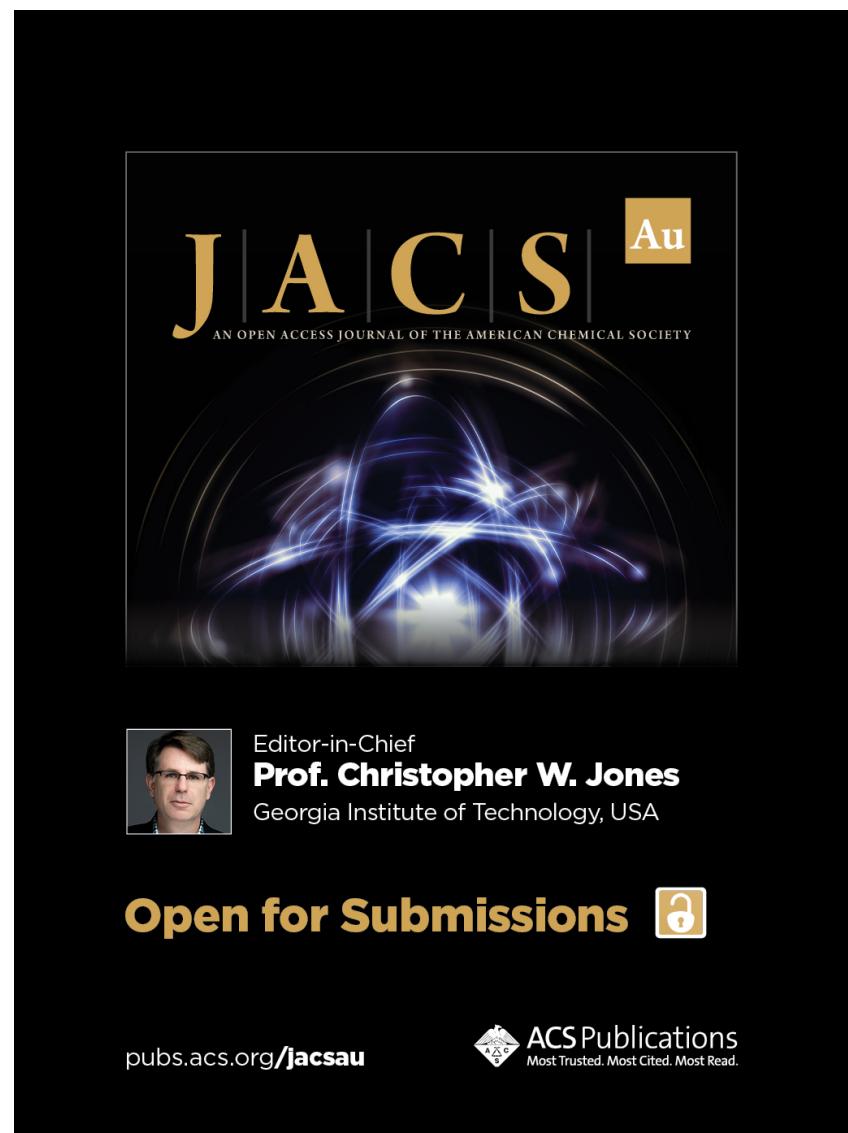

